\title{
Induced expression of precursor, mature and unprocessed forms of BDNF in the rat neonatal cortex
}

\author{
Shaburova E. ${ }^{1,2 *}$, Lanshakov D. ${ }^{1}$ \\ ${ }^{1}$ Institute of Cytology and Genetics, SB RAS, Novosibirsk, Russia \\ ${ }^{2}$ Novosibirsk State University, Novosibirsk, Russia \\ *e-mail: sliza.nsk@gmail.com
}

Key words: BDNF, dexamethasone, natural antisense BDNF, viral transduction

Motivation and Aim: We have previously found that even a single injection of dexamethasone on the third day of life led to changes in behavior and enhanced expression of $B d n f$ mRNA in the rat cortex within 5 days after the injection [1]. Changes in the expression of processed and non-processed forms of BDNF that have opposite effects on the formation of the neonatal cortex can be a possible reason for these hormone's effects. Methods and Algorithms: To test this hypothesis, we created lentiviral vectors which contain different cDNA: normal proBDNF, mBDNF without propeptide sequence, mutant mutBDNF, which is not processed due to mutation of the propeptide cleavage site, and control without insertion. These TET-ON-controlled vectors were injected into the cortex of rat pups on the first postnatal day. Expression of the vectors was activated by doxycycline treatment from 3rd to 8th days of life. After the 8th day, the material was taken to test DOX induction of expression with RT-PCR analysis. Remaining rats were raised without doxycycline up to one month. Statistical differences were determined by one-way or two-way ANOVA followed by Fisher's least significant difference post hoc analysis.

Results: On the 8th day of life, in the animals with the injection of mBDNF or mutBDNF vector, level of $B d n f$ mRNA expression in the cortex was significantly doubled, compared to control. At the same time, there was no increase in this level after the injection of proBDNF vector.

Conclusion: To explain the obtained results, a new hypothesis was proposed. There is a mechanism of $B d n f$ mRNA suppression by antisense RNA, which have a region of complementarity with the sequence encoding the propeptide. This mechanism is able to function only with the presence of proBDNF cDNA. Later, similar antisense RNA was found in humans and mice [2]. Our results suggest the existence of such a mechanism of $B d n f$ expression regulation in rats. Identification of the sequence of this antisense RNA in rats elucidates the functioning of this mechanism in this species and its participation in the development of the psychophysiological properties of the individual in early ontogenesis.

Acknowledgements: Supported by the RFBR (16-34-60103).

\section{References}

1. Bulygina V. et al. Dexamethasone upregulates levels of proapoptotic proteins proBDNF and cleaved caspase-3 in the frontal cortex and the hippocampus of neonatal rats. European Neuropsychopharmacol. 2017;27(4):S609-S610.

2. Modarresi F. et al. Inhibition of natural antisense transcripts in vivo results in gene-specific transcriptional upregulation. Nature Biotechnol. 2012;30(5):453. 